\title{
Mechanochemical Synthesis of Inorganic Halide Perovskites: Evolution of Phase-purity, Morphology, and Photoluminescence
}

Received 00th January 20xx, Accepted 00th January 20xx

\author{
Francisco Palazon, ${ }^{a, b *}$ Yousra El Ajjouri, ${ }^{a}$ Paz Sebastia-Luna, ${ }^{a}$ Simone Lauciello, ${ }^{c}$ Liberato Manna, ${ }^{b}$ \\ and Henk J. Bolink ${ }^{a}$
}

DOI: $10.1039 / x 0 x x 00000 x$

Dry mechanochemical ball-milling of halide precursor salts is a promising route for the synthesis of high-purity halide perovskites in a fast and solvent-free manner. However, there is a lack of information on the process mechanisms, kinetics, and possible side-effects. Here, we investigated in detail the mechanochemical synthesis of fully-inorganic $\mathrm{CsPbr}_{3}$ by ball-milling of stoichiometric $\mathrm{CsBr}$ and $\mathrm{PbBr}_{2}$. Detailed structural, morphological and optical analyses reveal several beneficial and detrimental effects of milling as a function of time. Three stages are identified during the process: (i) At short milling times ( $\mathrm{t}<5 \mathrm{~min}$ ) different ternary compounds are formed, including stoichiometric $\mathrm{CsPbBr}_{3}$ as well as $\mathrm{Cs}_{4} \mathrm{PbBr}_{6}$, and to a lesser extent, $\mathrm{CsPb}_{2} \mathrm{Br}_{5}$. Photoluminescence from "nano" and "bulk" $\mathrm{CsPbBr}_{3}$ species is observed, centered at $525 \mathrm{~nm}$ and $545 \mathrm{~nm}$, respectively. (ii) At the optimum time (around $5 \mathrm{~min}$ for the present case) the complete transformation of all reactants and byproducts into phase-pure $\mathrm{CsPbBr}_{3}$ has occurred. Photoluminescence corresponds to bulk $\mathrm{CsPbBr}_{3}$; (iii) At much longer milling times (up to 10 hours) eventually smaller quantum-confined $\mathrm{CsPbr}_{3} \mathrm{NCs}$ are exfoliated from the bulk product leading to a broad and blueshifted emission. At this stage the photoluminescence intensity is strongly reduced which is ascribed to the formation of surface defects induced by ball-milling in dry conditions.

One decade after their first use in photovoltaics, ${ }^{1}$ lead halide perovskites (LHPs) have become very promising materials for a wide range of optoelectronic applications such as solar cells ${ }^{2}$ and lightemitting diodes (LEDs). ${ }^{3}$ Thus, new ways of producing high-purity LHPs in large scale are actively sought after. Mechanochemical synthesis (MCS) has recently emerged as a very convenient and reliable method to obtain high quality LHPs as well as other lead-free multinary metal halides. ${ }^{4,5,14-23,6-13}$ Bulk materials have been obtained with a variety of compositions including fully-inorganic as well as hybrid organic-inorganic perovskites. MCS has been used to investigate the role of additives and dopants introduced in wellcontrolled small amounts. ${ }^{4,21,24,25}$ Colloidal quantum dots with photoluminescence quantum yields over $80 \%$ have also been

\footnotetext{
a. Instituto de Ciencia Molecular, Universidad de Valencia, C/ Catedrático J. Beltrán 2, 46980 Paterna, Spain.

b. Nanochemistry Department and ${ }^{C}$ Electron Microscopy Facility, Istituto Italiano di Tecnologia (IIT), Genova, Italy.

Electronic Supplementary Information (ESI) available: Experimental details and XRD. See DOI: 10.1039/x0xx00000x
}

produced by MCS.9,17,20,24,26 Eventually, ternary nonperovskite metal halides such as guanidinium-based $2 \mathrm{D}$ and $1 \mathrm{D}$ structures $^{19}$ or bismuth-based $2 \mathrm{D}$ and $\mathrm{OD}$ crystals ${ }^{22}$ have also been produced and shown to possess promising optoelectronic properties by MCS. It is also worth noting that materials prepared by MCS can be turned into thin films by solution- or vacuum-deposition techniques, thus enabling their use in common thin-film-based devices (e.g., solar cells or LEDs). ${ }^{5,6,8,11,15,18,24,26-28}$ All of these recent publications highlight the scientific and technological relevance of MCS for the study and production of LHPs and related materials. Nonetheless, there is scarce data in literature describing the process in detail. Little is known about the reaction kinetics, the formation of intermediate species (including impurities) and the effects of ball-milling on the size and shape of the final particles. The same is true about the formation of defects that can affect the optoelectronic properties of the material formed. To shed a new light on these issues, we carry out a systematic investigation of the mechanochemical synthesis of inorganic $\mathrm{CsPbBr}_{3}$ by dry ball-milling of stoichiometric $\mathrm{CsBr}: \mathrm{PbBr}_{2}$ mixtures. We evaluate the crystallinity, morphology and optical properties of the formed materials as a function of ball-milling time. Because we carried out this study without any solvents or additives, in stoichiometric conditions and with well-fixed parameters in a ballmill (i.e., not by hand grinding, where applied forces can be more variable) we expect that our results are symptomatic for the mechanochemical synthesis of halide perovskites in general.

To study the initial stages of the synthesis, stoichiometric $\mathrm{CsBr}: \mathrm{PbBr}_{2}$ mixtures were ball-milled for $0.5,1,2,3,4$, and 5 minutes. High-resolution PXRD data were acquired for all samples in the range $2 \Theta=10^{\circ}$ to $2 \Theta=90^{\circ}$ to identify and quantify the different species formed at each time. Aside from the binary precursors $\mathrm{CsBr}$ and $\mathrm{PbBr}_{2}$, three different ternary compounds are known to be stable: $\mathrm{CsPb}_{2} \mathrm{Br}_{5}, \mathrm{CsPbBr}_{3}$, and $\mathrm{Cs}_{4} \mathrm{PbBr}_{6}$. These phases are sometimes referred to as $2 \mathrm{D}, 3 \mathrm{D}$, and $\mathrm{OD}$, based on their structural dimensionality, that is, the interconnectivity of $\mathrm{PbBr}_{6}$ octahedra in the crystals. Figure $1 \mathrm{a}$ presents a close view of the $2 \Theta=10^{\circ}$ to $2 \Theta=$ $20^{\circ}$ range (see full patterns in Figure S1) where the three ternary compounds are clearly identified by peaks around $2 \Theta=11.7^{\circ}$ $\left(\mathrm{CsPb}_{2} \mathrm{Br}_{5}\right), 2 \Theta=12.7^{\circ}\left(\mathrm{Cs}_{4} \mathrm{PbBr}_{6}\right)$ and $2 \Theta=15.0^{\circ}\left(\mathrm{CsPbBr}_{3}\right)$, while $\mathrm{PbBr}_{2}$ is identified by a peak around $2 \Theta=18.5^{\circ}$. Note that these are not the main reflections of any of the phases. However, as they occur in a close yet not-overlapping region of the diffractogram, they are the most convenient for straightforward identification. As can be seen in Figure 1a, complete reaction into stoichiometric $\mathrm{CsPbBr}_{3}$ perovskite is achieved in less than 5 minutes. This highlights that 
simple dry ball-milling is an excellent technique to produce phasepure perovskites in a very fast and easy manner. Indeed, in view of these results it may seem that much longer times of several hours of ball-milling as reported by us and others previously might not be needed. ${ }^{15,20}$ However, we will discuss the effects of prolonged ballmilling later. Although high phase-purity is achieved in less than 5 minutes, non-stoichiometric $\mathrm{CsPb}_{2} \mathrm{Br}_{5}$ and $\mathrm{Cs}_{4} \mathrm{PbBr}_{6}$ is also present at intermediate stages, as previously discussed. It is worth noting that the $\mathrm{PbBr}_{2}$-rich phase $\mathrm{CsPb}_{2} \mathrm{Br}_{5}$ is only marginally seen in two of the six samples ( $<5 \%$ in all cases), while the $\mathrm{CsBr}$-rich phase $\mathrm{Cs}_{4} \mathrm{PbBr}_{6}$ is consistently observed in the all intermediate samples, reaching $>20 \%$ in weight. As the synthesis is carried out under stoichiometric conditions, this fact points towards a lower formation energy for $\mathrm{CsBr}$-rich phases. This is in agreement with DFT calculations which have estimated a formation energy of $-1.592 \mathrm{eV} /$ atom for $\mathrm{Cs}_{4} \mathrm{PbBr}_{6}$ and $-1.321 \mathrm{eV} /$ atom for $\mathrm{CsPbBr}_{3}{ }^{29,30}$

Furthermore, the fact that the three ternary phases co-exist at short milling times and eventually convert into phase-pure stoichiometric $\mathrm{CsPbBr}_{3}$ highlights the chemical and structural versatility of these compounds. Several reports have demonstrated interconversion between these phases. However, most of these were carried out in solution and/or with different additives such as amines, ${ }^{31} \mathrm{ZnX}_{2}{ }^{32}$ Prussian blue, $^{33}$ or water. ${ }^{34,35}$ Hence, it is worth noting that these interconversion reactions also take place during solid-state synthesis without any additive to mediate the reactions.

In order to obtain a semi-quantitative analysis of the different species, whole-pattern Le Bail fits were conducted (see Figure S1). ${ }^{36}$ Based on these, two quantification methods were used: On one hand, the calculated intensity of the main diffraction peak of each phase was divided by the relative intensity ratio (RIR) of the corresponding phase as obtained from literature (ICSD database). On the other hand, Rietveld refinements were conducted to directly obtain relative weight fractions from the structural model. Both methods resulted in nearly identical quantification results (see solid and dotted lines in Figure $1 \mathrm{~b}$-weight fraction as directly obtained from PXRD quantification- and Figure $1 \mathrm{c}$-molar fraction as derived from weight fraction and corresponding molecular weight of each phase-), despite slightly different pattern fits (Figure S1). Figure 1c shows that $\mathrm{CsBr}$ is consumed faster than $\mathrm{PbBr}_{2}$ as mostly $\mathrm{CsBr}$-rich phases are formed. In fact, between $t=1 \mathrm{~min}$ and $\mathrm{t}=2 \mathrm{~min}$ we observe a growth of the $\mathrm{Cs}_{4} \mathrm{PbBr}_{6} / \mathrm{CsPbBr}_{3}$ ratio. This highlights once again the versatile interconversion between the different ternary phases. The different reaction mixtures were also examined by scanning electron microscopy (SEM) and energy-dispersive $\mathrm{X}$-ray spectroscopy (EDX).
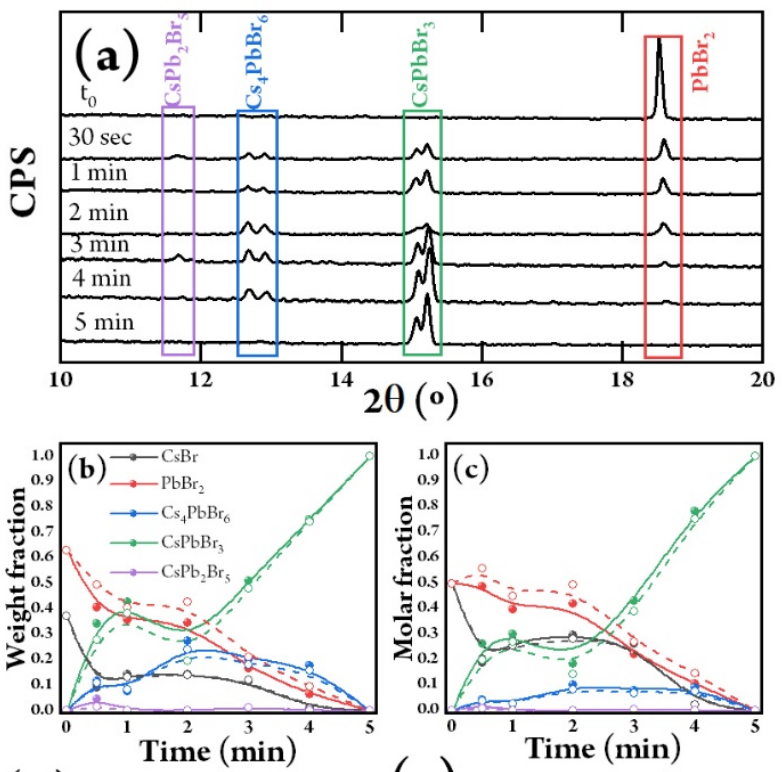

(d)

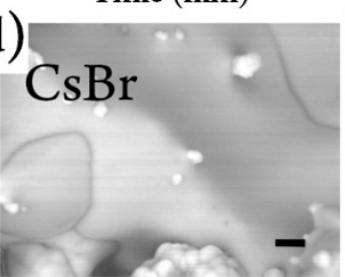

(e)
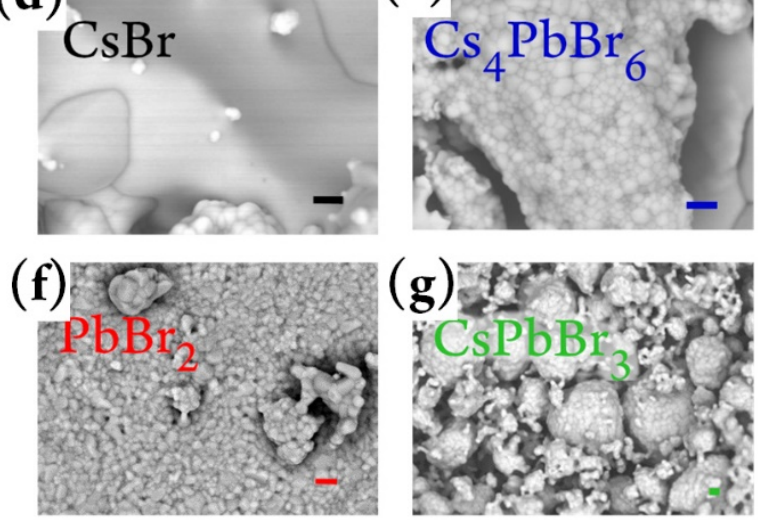

Figure 1. X-ray diffraction and scanning electron microscopy analysis of samples ballmilled for different times up to $5 \mathrm{~min}$. (a) Close view of the $2 \Theta=10^{\circ}$ to $2 \Theta=20^{\circ}$ range (see full patterns in Figure S1) of the diffractograms where characteristic peaks of different phases are highlighted in different colors. (b) Weight fraction of different species as derived from RIR method (filled spheres and solid lines) and Rietveld refinement (open circles and dashed lines). (c) Molar fraction of different species from RIR method (filled spheres and solid lines) and Rietveld refinement (open circles and dashed lines). B-spline lines are drawn to guide the eye. (d)-(g) Scanning electron microscopy of different phases found in the samples and identified by EDX analysis. All scale bars are one micron.

Figure $1 \mathrm{~d}$-g presents SEM images of the four main different compounds which are identified by EDX to be $\mathrm{CsBr}$ (49:51 ratio by EDX), $\mathrm{Cs}_{4} \mathrm{PbBr}_{6}$ (37:10:53 ratio by EDX), $\mathrm{PbBr}_{2}$ (33:67 ratio by EDX), and $\mathrm{CsPbBr}_{3}$ (22:18:60 ratio by EDX). These results are obtained by considering the high-energy lines for each element $(\mathrm{Br}(\mathrm{K}), \mathrm{Cs}(\mathrm{L})$, and $\mathrm{Pb}(\mathrm{L})$ ). The morphology of the different phases is clearly different: $\mathrm{CsBr}$ is formed of large smooth crystals while $\mathrm{PbBr}_{2}$ presents a rather small-grain texture. $\mathrm{Cs}_{4} \mathrm{PbBr}_{6}$ and $\mathrm{CsPbBr}_{3}$ consist of grains with different typical sizes from sub-100nm to $>1 \mu \mathrm{m}$. $\mathrm{CsPbBr}_{3}$ forms round-shaped aggregates of about 100 microns in size. Images presented in Figure 1d-g are selected close views of different phases identified by EDX. However, it is common to find large particles with different mixed phases. As an example, Figure 2 presents an SEM image of a large particle partly encapsulating fine-grain agglomerates. Corresponding EDX maps and selected small-area 
spectra show that the large particle is mostly $\mathrm{Pb}$-free $(\mathrm{CsBr})$ and the inside smaller grains are $\mathrm{CsPbBr}_{3}$.
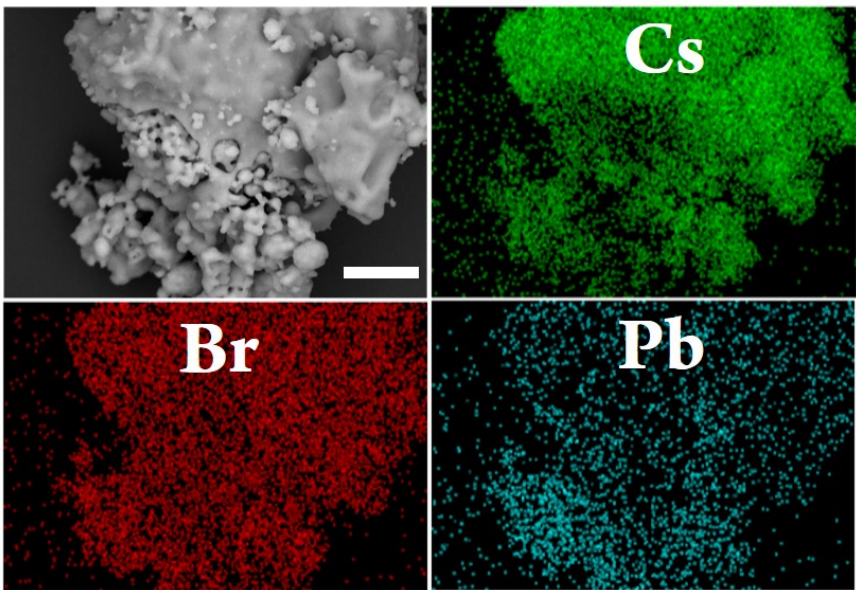

Figure 2. SEM image and corresponding EDX maps for $\mathrm{Cs}$ (green), $\mathrm{Br}$ (red), and $\mathrm{Pb}$ (blue). Scale bar is 10 microns. Two distinct materials are observed both from morphology and composition. These corresponds to $\mathrm{CsBr}$ (large smooth particle) and $\mathrm{CsPbr}_{3}$ (fine-grain agglomerates).

As mentioned before, several reports on mechanochemical synthesis of halide perovskites, including our own, ${ }^{15}$ use ball-milling times considerably longer than 5 minutes. Hereafter we investigated the effects of prolonged ball-milling for up to 10 hours. Diffractograms of samples ball-milled for $30 \mathrm{~min}, 1 \mathrm{~h}$ and $10 \mathrm{~h}$ can be fitted with a single $\mathrm{CsPbBr}_{3}$ phase (see Figure S2). This suggests that after full conversion into the stoichiometric $\mathrm{CsPBBr}_{3}$ phase, this perovskite 3D phase remains stable upon further ball-milling. This fact is not obvious $a$ priori, as we have previously seen that interconversion between the different ternary phases can happen. However, once full conversion into $\mathrm{CsPbBr}_{3}$ is achieved without remaining $\mathrm{PbBr}_{2}$ or $\mathrm{CsBr}$, it appears that no further structural evolutions take place under these conditions. In other words, $\mathrm{CsPbBr}_{3}$ is not reverted into its initial constituents $\mathrm{CsBr}+\mathrm{PbBr}_{2}$ upon ball-milling. Nonetheless, morphological changes are observed. The XRD peaks are broadened with prolonged MCS time (see Figure S3 for a qualitative assessment of line broadening). In order to study this in detail, microstructural analysis was performed on the whole-pattern fitted diffractograms (Figure S2). This was done after calibration to take into account instrumental broadening. Williamson-Hall plots (peak integral breadth vs inverse interatomic spacing) are presented in Figure 3a, where the slope is related to microstructural strain and the extrapolated value at origin gives an average isotropic grain size. For these analyses we discarded the possibility of anisotropy as no 1D (needle-) or 2D (platelet-) shaped crystals were observed from the SEM images (Figures $1 \mathrm{~g}$ and $\mathrm{S} 4$ ). For an easier evaluation the evolution of the apparent grain size is plotted in Figure $3 \mathrm{~b}$. A clear reduction in crystalline size is visible at longer milling times ( $t=10 \mathrm{~h})$. Nonetheless, it must be noted that this analysis provides an average size without information on size dispersity. Indeed, SEM images of the sample ball-milled for $10 \mathrm{~h}$ confirm the presence of small grains (sub-100nm) but also the coexistence with larger grains up to 1 micron (see Figure S4).
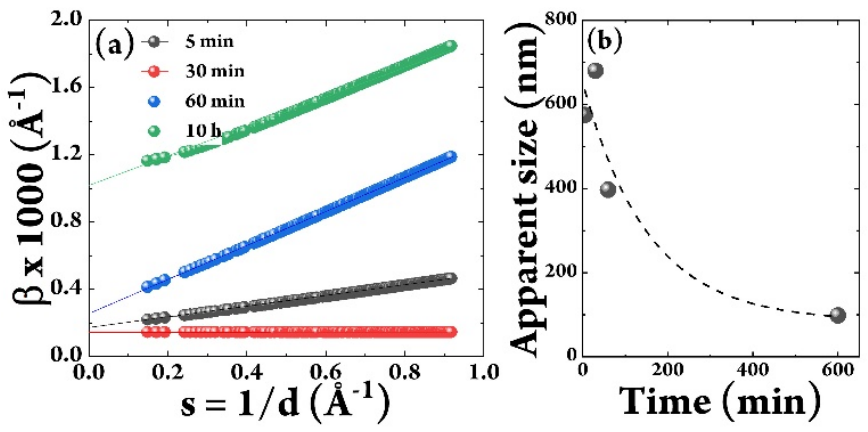

Figure 3. Microstructural XRD analysis. (a) Williamson-Hall (WH) plots obtained from Le Bail fits of XRD data acquired on samples ball-milled for $t=5$ min to $t=10 \mathrm{~h}$ (see Figure S3). Fits assume a pseudo-Voigt Thompson-Cox-Hastings ( $\mathrm{TCH}$ ) lineshape with gaussian broadening contribution from strain (slope) and lorentzian broadening contribution from reduced crystallite size (offset). (b) Apparent isotropic average crystal size deduced from extrapolated value at origin from WH plots.

To probe the evaluation of optoelectronic properties of the different powders the photoluminescence was determined by excitation with a UV laser $(\lambda=375 \mathrm{~nm}$ ) excitation (see Figure 4). Although these samples are generally not emissive enough to obtain reliable quantum yield measurements, we measured them in the same conditions (fixing the laser power, acquisition time, and sample amount) in order to get semi-quantitative results (Figure 3a). The first noticeable result is that the most-emissive samples are those that were ball-milled for short times $(t<5 \mathrm{~min})$, whereas the samples ball-milled for longer times demonstrated significantly lower PL intensities (one to two orders of magnitude lower). This reduction in $\mathrm{PL}$ intensity can be explained by the formation of non-radiative trap states resulting from defects induced by ball-milling. Besides the drop in PL intensity, a broadening of the spectra is observed (Figure 4b). In the first three samples ( $t=30 \mathrm{sec}, 1 \mathrm{~min}$, and $2 \mathrm{~min}$ ) which correspond to non-phase-pure synthesis as previously discussed, a dominant PL peak is observed centered at $525 \mathrm{~nm}$, with a second feature centered $545 \mathrm{~nm}$. These can be ascribed to weakly-quantumconfined (i.e., "nano") and bulk $\mathrm{CsPbBr}_{3}$. At $\mathrm{t}=5 \mathrm{~min}$, when the synthesis is complete, only a symmetric peak corresponding to bulk $\mathrm{CsPbBr} 3$ is present, with a narrow full-width at half maximum of 26 $\mathrm{nm}$. If further ball-milling is performed, aside from the alreadymentioned drop in PL intensity, the spectra become wider with a broad band at shorter wavelengths (Figure $4 \mathrm{~b}$ ). This can be ascribed to the exfoliation of small, quantum-confined $\mathrm{CsPbBr}_{3}$ nanocrystals. ${ }^{9}$ This observation is in agreement with the reduced average crystallite size revealed by microstructural XRD analysis (Figure 3 ). We note that exfoliation has been observed before upon prolonged ball-milling in the presence of solvents and ligand molecules. ${ }^{9}$ Our results suggest that these are not needed to obtain these smaller, quantumconfined crystals, although they are certainly beneficial to passivate their surfaces. This would appear critical to obtain high PL intensities as in our case, (by dry ball-milling without additives) the PL emission is greatly reduced. Eventually, it must be noted that optical absorption (Figure S5) is dominated by the lower-bandgap "bulk" species in all cases. 

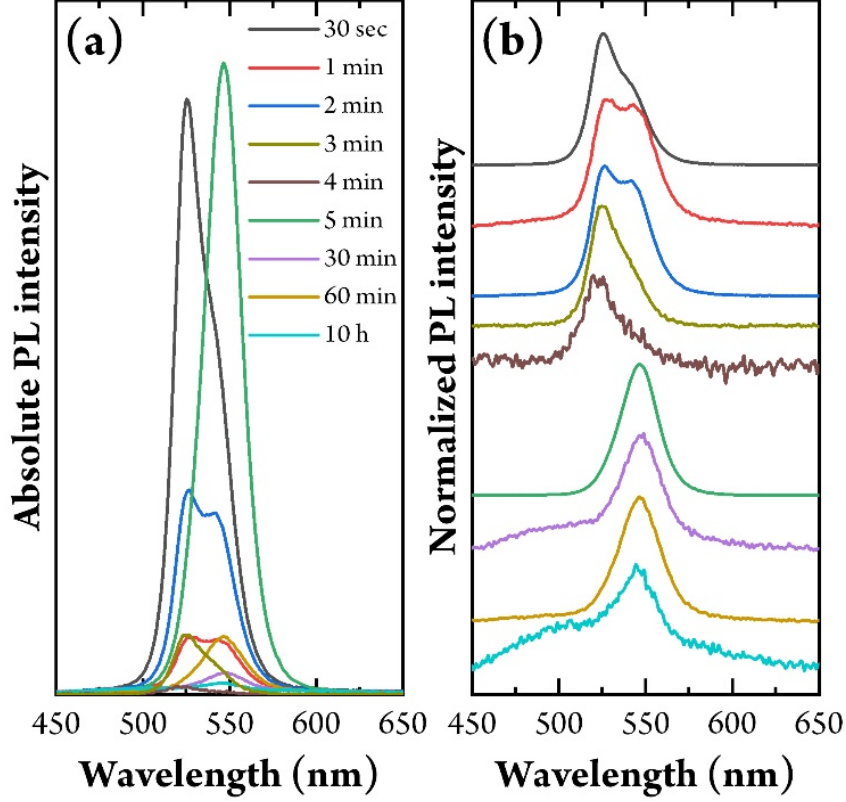

Figure 4. Absolute (a) and normalized (b) PL intensities of samples ball-milled for different times under identical measurement conditions (excitation source, acquisition time, and sample amount)

\section{Conclusions}

In summary, we followed over time the mechanochemical synthesis of an archetype halide perovskite, $\mathrm{CsPbBr}_{3}$, by dry ball-milling of stoichiometric precursors. An optimum ball-milling time exists (around $5 \mathrm{~min}$ for the material and ball milling system used; see experimental section for details) to obtain phase-pure 3D perovskites with narrow and intense PL emission. We believe it is important to underline that prolonged ball-milling, as has been used by us and others in the past leads to a broadening of the spectra and most importantly to an important loss of PL. From a more fundamental point of view, we have demonstrated the coexistence and interconversion of the three known ternary phases: $\mathrm{Cs}_{4} \mathrm{PbBr}_{6}$, $\mathrm{CsPbBr}$, and $\mathrm{CsPb}_{2} \mathrm{Br}_{5}$ at short milling times. In particular, we note that the $\mathrm{CsBr}$-rich phase is particularly important in the three samples obtained after ball-milling at short times. This may be associated to a lower formation energy for this compound. It is important to conclude that such a simple, fully dry, and fast (5 min) process leads to excellent phase-purity of fully-inorganic lead halide perovskites. This paves the way to a more generalized use of mechanochemistry for the synthesis of halide perovskites.

\section{Conflicts of interest}

There are no conflicts to declare.

\section{Acknowledgements}

The research leading to these results has received funding from the European Union Programme for Research and Innovation Horizon 2020 (2014-2020) under the Marie Skłodowska-Curie Grant Agreement PerovSAMs No. 747599, the Spanish Ministry of Economy and Competitiveness (MINECO) via the Unidad de Excelencia María de Maeztu MDM-2015-0538, MAT2017-88821-R and PCIN-2015255, and the Generalitat Valenciana (Prometeo/2016/135 and
GRISOLIAP/2017/089). F.P. thanks Javier Castells from Universidad de Valencia and Marine Reynaud from $\mathrm{CIC}$ energiGUNE for guidance in the use of Fullprof suite.

\section{References}

1 A. Kojima, K. Teshima, Y. Shirai and T. Miyasaka, J. Am. Chem. Soc., 2009, 131, 6050-6051.

A. K. Jena, A. Kulkarni and T. Miyasaka, Chem. Rev., 2019 119, 3036-3103.

X. Zhao, J. D. A. Ng, R. H. Friend and Z. K. Tan, ACS Photonics, 2018, 5, 3866-3875.

4 Y. El Ajjouri, V. S. Chirvony, M. Sessolo, F. Palazon and H. J. Bolink, RSC Adv., 2018, 8, 41548-41551.

5 D. Prochowicz, P. Yadav, M. Saliba, D. J. Kubicki, M. M. Tavakoli, S. M. Zakeeruddin, J. Lewiński, L. Emsley and M. Grätzel, Nano Energy, 2018, 49, 523-528.

O. Y. Posudievsky, N. V. Konoshchuk, V. L. Karbivskyy, O. P. Boiko, V. G. Koshechko and V. D. Pokhodenko, Theor. Exp. Chem., 2017, 53, 235-243.

J. Breternitz, S. Levcenko, H. Hempel, G. Gurieva, A. Franz, A. Hoser and S. Schorr, J. Phys. Energy, 2018, 1, 25003. O. Y. Posudievsky, N. V. Konoshchuk, A. G. Shkavro, V. L. Karbivskiy, V. G. Koshechko and V. D. Pokhodenko, ACS Appl. Nano Mater., 2018, 1, 4145-4155.

9 S. Yun, A. Kirakosyan, S. G. Yoon and J. Choi, ACS Sustain. Chem. Eng., 2018, 6, 3733-3738. M. Wilke and N. Casati, Chem. - A Eur. J., DOI:10.1002/chem.201804066.

11 D. Prochowicz, P. Yadav, M. Saliba, M. Saski, S. M. Zakeeruddin, J. Lewiński, M. Grätzel, M. Saliba, M. Saski, S. M. Zakeeruddin, J. Lewiński, M. Grätzel, M. Saliba, M. Saski, S. M. Zakeeruddin, J. Lewiński, M. Grätzel, M. Saliba, M. Saski, S. M. Zakeeruddin, J. Lewiński and M. Grätzel, Sustain. Energy Fuels, 2017, 1, 1-5.

12 P. Pal, S. Saha, A. Banik, A. Sarkar and K. Biswas, Chem. - A Eur. J., 2018, 24, 1811-1815.

13 A. M. Askar, A. Karmakar, G. M. Bernard, M. Ha, V. V. Terskikh, B. D. Wiltshire, S. Patel, J. Fleet, K. Shankar and V. K. Michaelis, J. Phys. Chem. Lett., 2018, 9, 2671-2677. A. Karmakar, A. M. Askar, G. M. Bernard, V. V. Terskikh, M. Ha, S. Patel, K. Shankar and V. K. Michaelis, Chem. Mater., 2018, 30, 2309-2321.

Y. El Ajjouri, F. Palazon, M. Sessolo and H. J. Bolink, Chem. Mater., 2018, 30, 7423-7427.

D. J. Kubicki, D. Prochowicz, A. Hofstetter, S. M.

Zakeeruddin, M. Grätzel and L. Emsley, J. Am. Chem. Soc., 2018, 140, 7232-7238.

17 Z.-Y. Y. Zhu, Q.-Q. Q. Yang, L.-F. F. Gao, L. Zhang, A.-Y. Y. Shi, C.-L. L. Sun, Q. Wang and H.-L. L. Zhang, J. Phys. Chem. Lett., 2017, 8, 1610-1614.

18 D. Prochowicz, M. Franckevičius, A. M. Cieślak, S. M. Zakeeruddin, M. Grätzel and J. Lewiński, J. Mater. Chem. A, 2015, 3, 20772-20777.

19 A. D. Jodlowski, A. Yépez, R. Luque, L. Camacho and G. de Miguel, Angew. Chemie - Int. Ed., 2016, 55, 14972-14977. L. Protesescu, S. Yakunin, O. Nazarenko, D. N. Dirin and M. V. Kovalenko, ACS Appl. Nano Mater., 2018, 1, 1300-1308.

21 D. J. Kubicki, D. Prochowicz, A. Hofstetter, S. M. Zakeeruddin, M. Grätzel and L. Emsley, J. Am. Chem. Soc., 
2017, 139, 14173-14180.

22 Y. El Ajjouri, V. S. Chirvony, N. Vassilyeva, M. Sessolo, F. Palazon and H. J. Bolink, J. Mater. Chem. C, , DOI:10.1039/C9TC01765H.

23 Y. El Ajjouri, F. Locardi, M. C. Gélvez-Rueda, M. Prato, M. Sessolo, M. Ferretti, F. C. Grozema, F. Palazon and H. J. Bolink, Energy Technol., 2019, ente.201900788.

24 W. Xiang, Z. Wang, D. J. Kubicki, W. Tress, J. Luo, D. Prochowicz, S. Akin, L. Emsley, J. Zhou, G. Dietler, M. Grätzel and A. Hagfeldt, Joule, 2019, 3, 205-214.

25 M. M. Tavakoli, W. Tress, J. V. Milić, D. Kubicki, L. Emsley and M. Grätzel, Energy Environ. Sci., 2018, 11, 3310-3320. D. Chen, J. Li, X. Chen, J. Chen and J. Zhong, ACS Appl. Mater. Interfaces, 2019, 11, 10059-10067.

27 D. J. Kubicki, D. Prochowicz, A. Hofstetter, M. Saski, P. Yadav, D. Bi, N. Pellet, J. Lewiński, S. M. Zakeeruddin, M. Grätzel and L. Emsley, J. Am. Chem. Soc., 2018, 140, 33453351.

28 B. Dou, L. M. Wheeler, J. A. Christians, D. T. Moore, S. P. Harvey, J. J. Berry, F. S. Barnes, S. E. Shaheen and M. F. A. M. Van Hest, ACS Energy Lett., 2018, 3, 979-985. K. Persson, , DOI:10.17188/1199510.

K. Persson, .

F. Palazon, G. Almeida, Q. A. Akkerman, L. De Trizio, Z. Dang, M. Prato and L. Manna, Chem. Mater., 2017, 29, 4167-4171.

32 S. K. Sharma, S. Mamgain, B. Attarwala and A. Yella, Nanoscale Adv., 2019, 00, 1-8.

33 F. Palazon, C. Urso, L. De Trizio, Q. Akkerman, S. Marras, F. Locardi, I. Nelli, M. Ferretti, M. Prato and L. Manna, ACS Energy Lett., 2017, 2445-2448.

34 X. Yu, L. Wu, H. Hu, M. Chen, Y. Tan, D. Yang, Q. Pan, Q. Zhong, T. Supasai and Q. Zhang, Langmuir, 2018, 6, acs.langmuir.8b01683.

35 L. Wu, H. Hu, Y. Xu, S. Jiang, M. Chen, Q. Zhong, D. Yang, Q. Liu, Y. Zhao, B. Sun, Q. Zhang and Y. Yin, Nano Lett., 2017, 6, acs.nanolett.7b02896.

36 A. Le Bail, Powder Diffr., 2005, 20, 316-326. 